\title{
SHAFER-FINK TYPE INEQUALITIES FOR THE ELLIPTIC FUNCTION $\operatorname{sn}(\mathbf{u} \mid \mathbf{k})$
}

\author{
A. MCD. MERCER
}

Abstract. The inequalities of Shafer and Fink, namely,

$$
\frac{3 x}{2+\sqrt{1-x^{2}}} \leqslant \sin ^{-1}(x) \leqslant \frac{\pi x}{2+\sqrt{1-x^{2}}}, \quad x \in[0,1)
$$

are generalized to similar inequalities for the elliptic function $\operatorname{sn}(u \mid k)$.

Mathematics subject classification (2000): 26D05.

Key words and phrases: Shafer-Fink inequalities, Jacobi elliptic function.

\section{REFERENCES}

[1] R. E. SHAFER, Problem E1867, Amer. Math. Monthly, 74 (6) (1967), 726-727.

2] A. M. Fink, Two Inequalities, Univ. Beograd. Pub. Elekt. Fak., 6 (1995), 48-49.

[3] Ling ZHU, On Shafer-Fink-Type Inequalities, J. Ineq. Appl. Vol. (2007), Article ID 67430.

[4] MEYER, K. R., Jacobi elliptic functions from a dynamical systems point of view, Amer. Math. Monthly, 108 (8) (0ctober 2001), 729-737.

[5] Wolfram MathWorld, URL: http://mathworld.wolfram.com/JacobiEllpticFunctions.html

[6] G. T. WhitTAKER, G. N. WATsOn, A Course of Modern Analysis, Cambridge University Press, Cambridge (1952). 